\title{
Reward systems and cognitions in Major Depressive Disorder
}

\author{
Marie-Laure Cléry-Melin, ${ }^{1,2}$ Fabrice Jollant, ${ }^{1,2}$ and Philip Gorwood ${ }^{1,2} *$
}

\author{
${ }^{1}$ CMME, Hôpital Sainte-Anne, Université Paris Descartes, 100 rue de la Santé, 75014 Paris, France \\ ${ }^{2}$ Centre de Psychiatrie et Neuroscience (INSERM UMR 894), 2 ter rue d'Alésia, 75014 Paris, France
}

\begin{abstract}
A lack of motivation and anhedonia represent frequent and pervasive symptoms in depression, although with poor specificity. Historically described as a response bias, reward-related impairments in depression may account for the important aspects of the cognitive impairments associated with diagnosis of major depressive disorder. Reward processing is a broad psychological construct that can be parsed into 3 distinct components known as "reinforcement learning" (learning), "reward responsiveness" (liking), and "motivation to obtain a reward" (wanting). Depressed patients respond hyposensitively to reward and maladaptively to punishment: this pattern is related to a dysfunction in the frontostriatal systems modulated by the monoamine systems; seems to be observed in medicated and unmedicated patients with depression and in healthy individuals with high levels of anhedonia; and could be observed in patients with a history of depression, even when in full remission. Considered to be cognitive impairments, reward-relatedimpairments may also constitute part of an underlying neurobiological vulnerability to major depressive disorder (MDD). For example, the reward-related impairment is state dependent and, more or less, correlated with symptom severity in some studies but has also been proposed as being trait like, with endophenotype characteristics, possibly contributing to the persistence of the disease or treatment resistance. The 3 core aspects of reward processing have specific neurobiological correlates that involve the ventral and dorsal striatum, lateral habenula, ventral tegmental area, orbitofrontal cortex, anterior cingulate cortex, and ventromedial and dorsolateral prefrontal cortex. These structures underline the important role of the dopaminergic mesolimbic pathway, but glutamate and serotonin could also have an important role, at least in some aspects of reward-related impairments.
\end{abstract}

Received 23 January 2018; Accepted 28 August 2018; First published online 26 November 2018

Key words: anhedonia, cognition, dopamine, endophenotype, liking, motivation, neurotoxicity, reward, striatum, wanting.

\section{Introduction}

Diagnosis with major depressive disorder (MDD) primarily involves either a depressed mood or a loss of interest or pleasure in most usual activities most of the day, nearly every day, according to the DSM-5. ${ }^{1}$ Rewardrelated symptoms such as a lack of motivation and anhedonia represent frequent and pervasive symptoms in depression, as in other neuropsychiatric diseases. Historically described as a "response bias" (bias that influences the response of participants to a stimulus away from an accurate response, irrespective of stimulus presentation), "negative cognitive set," or "disruptions in arousal-activation," reward-related impairments in depression interfere with cognitive performance, ${ }^{2}$

\footnotetext{
* Address correspondence to: Professor Philip Gorwood, MD, PhD, Sainte Anne Hospital, Paris, FRANCE.

(Email: p.gorwood@ch-sainte-anne.fr)
}

contribute to difficulties encountered in performing cognitive effortful tasks ${ }^{3}$ and decision-making, and, thus, may account for cognitive impairments. Other more specific cognitive symptoms are equally integral to the diagnosis of MDD, such as a diminished ability to think or concentrate, indecisiveness, or psychomotor retardation. ${ }^{1}$ Depression-related impairments in attention, memory, psychomotor speed, and executive functioning that were assessed by laboratory tasks are well described at the acute phase of a major depressive episode (MDE), might persist beyond clinical recovery, are related to neuroimaging abnormalities, and could have trait-like characteristics. ${ }^{4}$ Such impaired cognitive processes are likely to contribute to reward-related impairments assessed in depression.

However, there is a growing body of evidence suggesting that impaired ability to use information (ie, the cost and benefits or the magnitude and probability of 
rewards) to expend an effort and to guide or adapt their choice behaviors are crucial cognitive aspects of rewardrelated symptoms in depressed patients. ${ }^{5}$ Research is necessitated to obtain a comprehensive understanding of the underlying neuropsychological and neurobiological mechanisms of both reward-related and cognitive impairments, as they seem to coexist in depressed patients, even after remission, and lead to a higher risk of poorer treatment response, functional outcome, and relapse or recurrence. $^{6-9}$

The present review aims to analyze reward processes and cognitions impaired in MDD and their characteristics and neurobiological substrates to provide better assessments, prevention, and treatment strategies. A literature search was initially conducted in the PubMed (Medline) database, to identify articles with the following key words: "reward" or "reward system" and "cognitive" or "cognition" and "depressive disorder" or "depression" not "bipolar" not "addiction" and not "schizophrenia"). Some articles that did not correspond to the topic of interest were excluded after screening the abstracts, and some were included after referencing retrospectively from the initially selected articles and additional ad hoc searches.

We focused on reward-related processes that are considered by many behavioral studies as cognitive processes and equally impaired in depression, as in suicidal behavior. Reward-related impairment may constitute possible endophenotypes of depression, ie, simpler phenotypes used to aid in characterizing the disorder and its underlying substrates. ${ }^{10} \mathrm{~A}$ better understanding of the neural network subserving the rewardrelated impairments in depression would help to characterize the disease better and potentially provide better assessments and preventive and therapeutic strategies to reduce reward-related impairments.

\section{Cognitive Behavioral Studies of Reward-Related Processes in MDD}

\section{Focus on reward-related processes as cognitive processes}

Reward processing is a broad psychological construct that can be parsed into 3 distinct neuropsychological sub-components known as "reward-related learning" or "reinforcement learning," "hedonic capacity," and "motivation to obtain a reward." These reward-related processes play key roles in optimizing the allocation of brain resources necessary for evolutionary survival and for maintaining spontaneous activity, by helping to drive behavior adaptively among different options. They may be supported by partially dissociable brain systems according to neuroimaging findings and underlie dysfunctional reward-related behaviors in depression. ${ }^{11-13}$

Reward-related learning (or reinforcement learning) consists of the ability to represent the rewarding (or punishing) value of a stimulus and, then, to establish and update predictions of future reward to guide and modify behavior (with the highest and well-estimated overall predicted reward value) to maximize reward and/or minimize punishment. ${ }^{14}$ The subject may either exploit a previously learned model (model-based learning) or rely on some trial and error experience (model-free learning) for action selection. ${ }^{6}$ Value prediction is usually assessed in classical conditioning involving signal detection (eg, probabilistic reward task $[\mathrm{PRT}]^{15}$ ) or decision-making paradigms (eg, Iowa Gambling Task $\left.[\mathrm{IGT}]^{8}\right)$. They measure the ability to learn from reward feedback and involve a large cerebral network underlying cognitive and reward processing functions (striatum and frontocingulate regions). ${ }^{6,16}$

Hedonic capacity (or reward sensitivity, reward responsiveness) is another reward-related process explored by examining "liking" reactions (eg, ratings or behavioral reactions toward pleasantness ${ }^{13}$ ) that capture some aspect of pleasure and mainly involve limbic structures (the nucleus accumbens and ventral pallidum). ${ }^{12}$ The degree of response bias (propensity to select one or the other response irrespective of stimulus presentation), for example, in signal detection tasks, can also be used to objectively assess reward responsiveness (such as a preference for the response paired with a more frequent reward). ${ }^{15}$

Finally, motivation to obtain a reward has been linked to the concept of "wanting" and is also called incentive salience, a type of incentive motivation that does not always require elaborate explicit expectations of future outcomes. Motivation is focused more directly on reward-related (innate or learned) stimuli and involves subcortical neural systems that include mesolimbic dopamine projections. Research has established that "wanting" and "liking" rewards, as well as "wanting" and reward learning (association and prediction), are dissociable psychologically and neurobiologically. ${ }^{12}$ In studies recently performed and reviewed by Pessiglione et al, ${ }^{17}$ motivation is defined as the function that orients and activates the behavior to achieve a goal and assessed by investigating the trade-off between the expected cost entailed by potential actions (mostly focusing on the amount of effort the subject is willing to expend) and the expected benefit associated with potential rewards. Different types of tasks have been developed over the past decade, commonly known as effort-cost decisionmaking (ECDM) tasks. Effort-based decision tasks (eg, binary choice such as the button-pressing effort expenditure for rewards task [EEfRT] ${ }^{5,18,19}$ ), incentive motivation tasks (eg, willingness to accept or decline performing a single action ${ }^{20}$ ) and free-operant tasks (eg, selection within a continuous range such as a handgrip force measurement squeezing $\operatorname{task}^{21}$ ) are specific behavioral tasks quantifying motivation as an 
effort/reward trade-off. The underlying effort and reward networks include the dorsal anterior cingulate cortex (dACG) and its connections to the anterior insula (aI) and premotor and motor areas and ventral frontostriatal circuits, as well as the dopaminergic nuclei, mainly involving serotoninergic, dopaminergic, and noradrenergic modulation ${ }^{17}$ (Figure 1).

In a recent review by Hélie et al, the authors assumed that reward processing and valuation (a process of assigning values to states and actions based on the subject's current representation of the environment occurring before action) are essential steps in any cognitive process and can even be considered cognitive functions, on which many other cognitive functions rely. ${ }^{7}$

In this view and considering findings on patients' deficits in processing information to guide behavior, many cognitive impairments observed in depression (among other neuropsychiatric disorders) are associated with or may be accounted for by deficits in reward processing. The terms reinforcement learning, reward responsiveness and motivation to obtain a reward will be the ones used throughout the manuscript for reasons of simplicity.

\section{Characterization of reward-related impairments in depression}

Research on reward processing in depression over the past decade first focused on behavioral responses to reward and punishment. Common findings, mainly obtained from reward reinforcement learning paradigms, indicated that depressed patients respond hyposensitively to a reward $\mathrm{d}^{11,22}$ and maladaptively to a punishment (worse performance demonstrating increased sensitivity to punishment and blunted responses to reinforcement). This pattern is related to a dysfunction in the frontostriatal systems modulated by the monoamine systems. ${ }^{23}$ Reinforcement learning is impaired in both medicated and unmedicated patients with depression (particularly those with high anhedonic symptoms) who fail to modulate behavior as a function of prior reinforcement or feedback in a probabilistic task. $^{6,15,24}$ They are also observed in healthy individuals with high levels of anhedonia. ${ }^{11}$ Interestingly, reduced reward learning in depressed patients before treatment onset was predictive of a persistent diagnosis of depression 8 weeks later, even when controlling for baseline depression severity and comorbid anxiety, and, thus, may contribute to the persistence of MDD or treatment resistance. ${ }^{24}$ Further, there is evidence for a persistent reward learning impairment in euthymic individuals with a history of depression in full remission, as compared with healthy controls, ${ }^{25,26}$ possibly attributable to abnormalities in the neural processes that support reward feedback monitoring (eg, reduced activity in the ACC). ${ }^{26}$

However, the underlying mechanisms remain unclear, as an impaired learning rate (ability to update an outcome value and using prediction errors information),

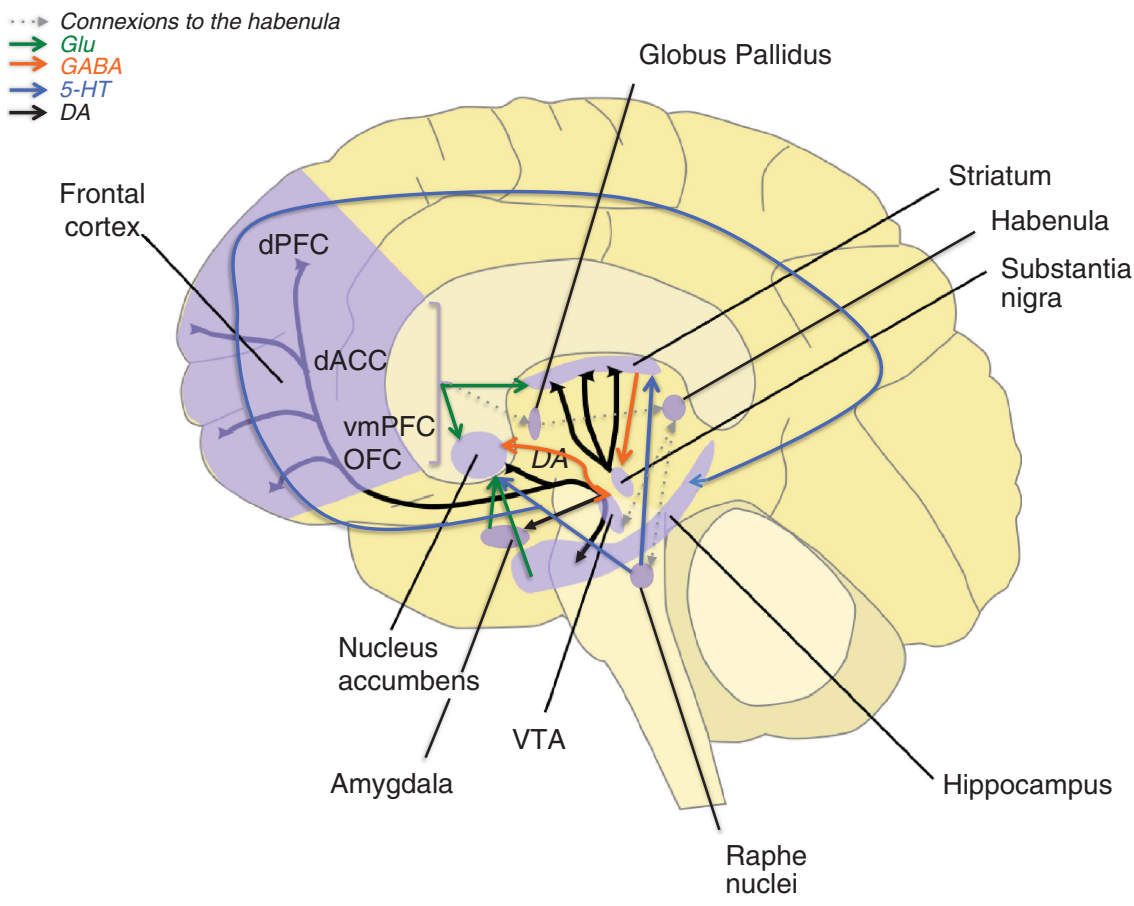

FIGURE 1. Reward-related processes: main regions and neurotransmitters 5-HT, serotonin; DA, dopamine; dACC, dorsal anterior prefrontal cortex; dPFC, dorsal prefrontal cortex; Glu, glutamate; GABA, gamma amino butyric acid; OFC, orbitofrontal cortex; vmPC, ventromedial prefrontal cortex; VTA, ventral tegmental area. 
in both reward and punishment, has inconsistently been detected in MDD patients, depending on several factors (ie, specific paradigms and patient characteristics). ${ }^{6,27}$ Moreover, in a meta-analysis of studies that implemented reinforcement learning tasks, Huys et al (2013) concluded that depression, as well as subclinical anhedonia in healthy controls, primarily affected reward responsiveness rather than learning rate. ${ }^{22}$

Reward responsiveness, reflecting hedonic capacity, might indeed be dysfunctional in depression and considered a potential contributory mechanism for depressive symptom. ${ }^{28,29}$ For example, patients with unmedicated MDD fail to express a response bias toward a more frequently rewarded stimulus (an indicator of reward responsiveness), this impairment being correlated with self-reported anhedonia. ${ }^{15}$ Using the same paradigm, lower reward response biases were also found in patients with MDD in full remission, as compared with healthy controls, indicating that a blunted reward responsiveness endures through the different stages of the disease and might be a trait-related abnormality in MDD. ${ }^{25}$ These findings are consistent with reduced striatal reactivity to reward outcomes, possibly associated with disrupted dopamine and opioid/endocannabinoid transmission. ${ }^{6,24}$

According to Pizzagalli et al, it may be assumed that a blunted reward responsiveness leads to decreased engagement in pleasurable activities and decreased motivational drive to obtain future reward. ${ }^{15}$ Thus, anhedonia, rather than a reduction in pleasure per se, would consist of an impaired ability to modify behavior as a function of reward or a dissociation between pleasure and the amount of effort expended to achieve it. ${ }^{11,30}$ In this view, a reduced reward responsiveness and its associated neural correlates could represent an interesting pathway driving effort deficits in depression. ${ }^{29}$

Recent findings have also provided compelling evidence of aberrant reward/effort-based decision-making in depression, contributing to motivational impairment. A first study using a handgrip device to assess incentive motivation showed that contrary to healthy controls, patients with MDD failed to increase effort expenditure when more money was at stake. ${ }^{31}$ In a follow-up study, patients after remission from MDE retrieved a normal sensitivity to incentives (ie, a normal pattern of effort production in response to monetary rewards), associated with an improvement in apathy scores. ${ }^{32}$ Similarly, studies using different ECDM paradigms (binary choice, willingness to accept, and handgrip squeezing tasks) have found consistent evidence for reduced willingness to expend effort to incentives in patients with MDD, as compared with controls. ${ }^{5,18,19,29,33,34}$ Decreased effort expenditure in depressed patients was correlated with increased self-reported anhedonic symptoms or lower reward expectancy, ${ }^{18,34}$ a longer duration of the current episode, ${ }^{5}$ and higher apathy scores. ${ }^{32}$ Interestingly, a correlation with depression severity scores (Beck Depression Inventory [BDI] scores), assessed in depressed, remitted, or control subjects, was contradictory among studies, ${ }^{18,33,35}$ suggesting that decreased effort expenditure might represent a specific dimension of depression rather than a consequence of depression severity ${ }^{17}$ and possibly have trait-like characteristics, as discussed below.

\section{Reward-Related Impairments as Vulnerability Markers of the Disease}

Some more specific cognitive dysfunctions, particularly for executive functioning, verbal learning, and memory assessed by neurocognitive tests, persist and somehow progress among clinically remitted and depressed patients, potentially representing trait markers of the disorder, even though the mechanisms involved are not yet fully understood. ${ }^{36,37}$ Considered to be cognitive impairments, a reward-related impairment may also constitute part of an underlying neurobiological vulnerability to MDD and, thus, have useful clinical implications.

\section{Characteristics of reward-related impairments as vulnerability markers}

Some studies, mostly studying the ECDM motivational component, support the hypothesis that reward-related impairments are state dependent and, more or less, correlated with symptom severity. ${ }^{18,32,38}$ However, more research on this aspect is required, as consistent findings indicate that they have trait-like, and even endophenotype, characteristics (Table 1). As described above, comprehensive studies showed that reward-related learning impairment at the acute stage of an MDE predicted treatment outcome (above and beyond baseline depression severity and anxiety comorbidity), possibly contributing to the persistence of the disease or treatment resistance. $^{24}$

Both reward reinforcement learning impairment and blunted reward responsiveness, often concurrently measured in signal detection tasks (eg, probabilistic reward task), are observed in acute MDD and endure when patients are in full remission, even years after the last $\mathrm{MDE},{ }^{25,26}$ possibly leading to disease recurrence.

Interestingly, consistent neuroimaging findings found abnormalities in reward-related brain activation in not only unmedicated recovered patients with depression but also young people at risk of depression, particularly in striatal or cortical regions playing a role in linking actions to positive and negative feedback (ACC, orbitofrontal cortex [OFC]). ${ }^{39,40}$ Moreover, in a prospective study conducted in never-depressed at-risk adolescents, 


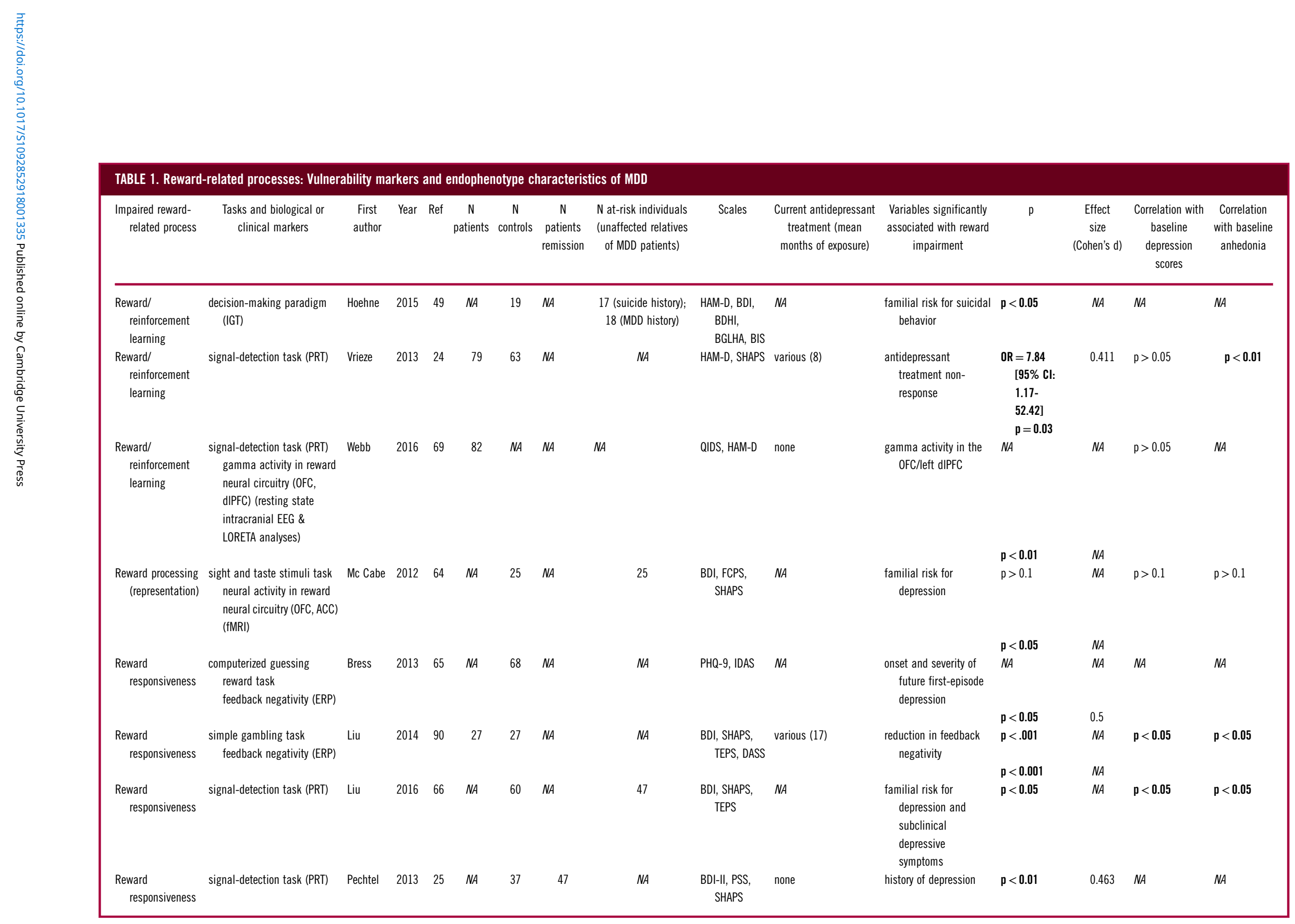




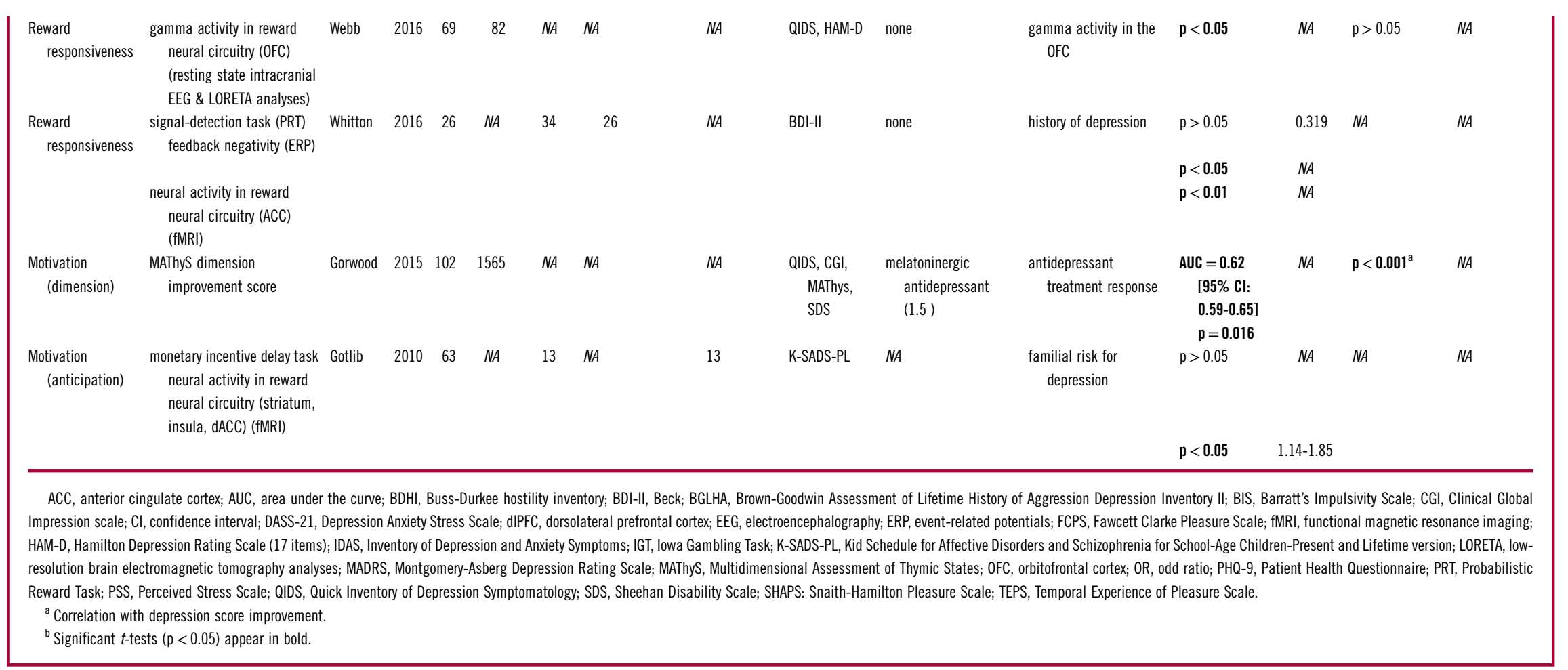


an impaired neural measure of reward responsiveness (using an event-related potential [ERP] component) at baseline predicted the subsequent onset of MDEs and the severity of future depressive symptoms. ${ }^{41}$ Finally, blunted reward responsiveness, associated with a high degree of anhedonia, was found in first-degree relatives of patients with MDD experiencing subclinical depressive symptoms, as compared with healthy controls. ${ }^{42}$

Reward-related processing impairments might be a trait marker related to a patient's vulnerability to depression, occurring before onset and throughout the different phases of the illness. Its independent expression beyond an acute MDE and its familial association led to consider these impairments as likely endophenotypes for MDD using the criteria of Gottesman and Gould..$^{10}$ As of 2004, Hasler et al considered an impaired reward function associated with anhedonia as a specific and plausible endophenotype of MDD and being related to a dysfunction in the brain reward system according to molecular, genetic, and epidemiological research. ${ }^{43}$ Anhedonia, as part of the reward-related impairments (reduced responsiveness to rewarding stimuli specifically) and risk factors for depression, has more recently been considered a promising endophenotype of depression. According to Pizzagalli (2014), the anhedonic behavior could be induced by the pervasive effects of stress (chronic stressors and early childhood adversities) on brain reward systems (specifically mesocorticolimbic dopaminergic pathways) implicated in reinforcement learning and incentive motivation. ${ }^{30}$ Interestingly, preliminary findings from a twin study indicated that reward responsiveness is heritable, with additive genetic factors contributing to $46 \%$ of the variance. ${ }^{44}$ Emerging findings also imply that blunted reward reinforcement learning is a promising endophenotype. ${ }^{26,45}$ According to Webb et al (2016), this reward-related component has emerged as the most promising behavioral endophenotypes of depression, together with neuroticism and cognitive control. $^{45}$ Blunted reward learning was significantly associated with reduced gamma activity in the left dorsolateral prefrontal cortex (dIPFC) and OFC (those regions also involved in reduced reward responsiveness) on resting-state intracranial EEG measurements, even while controlling for the current symptoms and task difficulty. In this study, the 3 putative endophenotypes had partially dissociable resting electroencephalography (EEG) correlates, reflecting underlying neural dysfunctions that will be further reviewed in the next section.

\section{Neurobiological correlates of reward-related processes}

Many neuroimaging studies using task-related functional magnetic resonance imaging (fMRI) have focused on activity changes in the regions and circuits involved in the reward processes in MDD (Figure 1) while performing reward-related tasks and found significant differences in patients with MDD, as compared with healthy controls.

First, these studies reported hyporesponsivity and decreased activation in the striatal regions, and their role in the different reward processing sub-components has already been shown, ${ }^{14,46}$ in both the ventral striatum (including the nucleus accumbens [NAc]) and dorsal striatum (caudate nucleus and putamen) in patients with depression. ${ }^{11,47}$

The NAc, as a main component of the dopaminergic mesolimbic pathway through its connections with the ventral tegmental area (VTA) and substantia nigra (SN), plays a central role in reward processes (anticipation and drive to obtain reward) and may even represent a promising target for deep brain stimulation (DBS) as an effective treatment for resistant MDD. ${ }^{48}$ In addition to the massive dopaminergic input from the midbrain (VTA/SN), the ventral striatum receives its main cortical input from the OFC and ACG. ${ }^{46}$ The OFC, together with the ventral striatum and amygdala, is involved in maintaining representations and predicting reward values, necessary to guide action selection for reward and adjust behavior. ${ }^{16,49,50}$ Neural processing of reward information (eg, discrepancies between expected and actual reward, namely prediction errors) is specifically attenuated in the medial OFC (mOFG) in unmedicated patients with MDD, as compared with healthy participants, even if their behavioral performances (reinforcement learning) does not significantly differ, possibly reflecting the specific role of this region in the ability to experience pleasure from positive outcomes. ${ }^{27}$ The ACC receives information from the $\mathrm{OFC}$ on the reward value and is involved in the evaluation of effort (cost)/reward (benefit) options to determine the effort required for possible actions. The ACC sends projections to the ventromedial prefrontal cortex (vmPFC) and dIPFC that are involved in decision-making based on reward value, effort, and reinforcement history regarding future actions. $^{26,51-53}$

Several findings on the dorsal striatum (caudate and putamen) indicate that it may be involved in stimulusresponse learning (while the ventral striatum is more involved in stimulus-reward learning), responding to the reinforcement of an action and linking action selection to predicted reward ${ }^{16,26,30,51-54}$ The lateral habenula $(\mathrm{LHb})$, a small structure located at the posterior end of the thalamus, within the neural reward circuitry, is coming under scrutiny in a growing number of studies. It is thought to be involved in the avoidance system of the brain, mainly through encoding negative reward prediction errors. Habenular hyperactivity may contribute to anhedonia and symptoms of depression related to reinforcement learning, such as abnormal response to 
punishment. ${ }^{11,55}$ The habenula, as the ventral striatum, represents a promising target for DBS. Even if further investigation is required, this treatment appears to provide significant clinical improvement in severely affected and refractory patients. ${ }^{56}$

Functional MRI studies have further suggested that reward-related impairments in depression are related to abnormal cortical-striatal activity and connectivity that may even predict antidepressant treatment outcomes in depression ${ }^{11,56,57}$ For example, in patients with depression, but not in healthy individuals, an acute pharmacological challenge inducing enhancement of dopaminergic transmission (using a single dose of the D2/D3 receptor antagonist, amisulpride) increased striatal activity and cortical-striatal functional activity in response to rewards. Besides, a stronger corticostriatal functional connectivity between the NAc and midcingulate cortex was associated with better reward learning performance. ${ }^{58}$

More recently, specific resting-state fMRI studies, investigating correlations in activity across different regions to delineate large-scale functional networks, have shown abnormal functional connectivity within and between networks (eg, Central Executive Network, Salience Network, Default-Mode Network), involved in reward-related cognitive deficits and depressive mood in patients with MDD. ${ }^{58-62}$ For example, compared with control subjects, patients with MDD had disrupted functional connectivity of the bilateral NAc networks primarily located in the prefrontal-striatal (OFG, ACG, and caudate) regions that was significantly associated with nonspecific cognitive deficits (Mini-Mental State Examination [MMSE] $)^{63}$ and depression severity. ${ }^{59}$ Interestingly, in a resting-state fMRI study exploring the whole connectome in relation to reward responsiveness across participants with different psychiatric diagnosis (including MDD), Sharma et al (2017) found that reward responsiveness deficits were associated with connectivity abnormalities between the NAc and distinct major cortical functional networks, regardless of clinical diagnosis category. ${ }^{64}$ Moreover, impairment in motivation to obtain a reward, assessed in an effort-based reinforcement task, was associated with deficient $\mathrm{mOFC}^{-}$ striatal functional connectivity, as compared with not only healthy controls but also patients with schizophrenia, suggesting that a common symptom such as motivational impairment may involve different prefrontal-striatal pathways in different psychiatric disorders. ${ }^{65}$

Electrophysiology studies have had interesting findings, using ERP to assess reward-related impairment in MDD. ERP studies have focused on feedback negativity (FN), an index of early evaluation of rewards, as compared with non-rewards, which is usually maximal at the frontocentral electrodes, $300 \mathrm{~ms}$ following reward feedback. There is converging evidence that an FN amplitude represents a neural measure of reward sensitivity, one of the main reward-related processes. FN amplitude is blunted in MDD, is related to anhedonia severity and depressive symptoms, ${ }^{66}$ and correlates with blunted ventral striatum activation especially in patients with melancholic features such as impaired mood reactivity. ${ }^{67}$ Moreover, a blunted FN amplitude at baseline in adolescent girls who have never had depression independently predicted the subsequent onset of the first episode and severity of symptoms during the follow-up period. $^{41}$

With regards to neurotransmitters, dopamine plays a critical role in reinforcement learning and incentive motivation. ${ }^{30}$ Dysfunction within the mesolimbic dopaminergic pathway projecting from the VTA to the ventral striatum (NAc) may have a key role in the pathophysiology of depression and particularly in reinforcement learning, as ventral striatal dopamine regulates the prediction and anticipation of rewards, the 2 mechanisms responsible for reinforcement learning. ${ }^{52,58}$ Recent evidence in animal and human studies implicated other neurotransmitters in reward processing, including glutamate, gamma-aminobutyric acid (GABA), and serotonin ${ }^{11,52,68}$ The NAc receives glutamatergic input from the amygdala, increasing motivation to achieve a goal-directed action planned in the prefrontal areas. Ketamine (N-methyl-D-aspartate [NMDA], or glutamate receptor antagonist) has an antidepressant effect that may be mediated by glutamatergic signaling, blocking glutamate uptake in the prefrontal cortex, resulting in reward impairment. ${ }^{69}$ Glutamatergic activity and also probably opioid activity in the amygdala are necessary for motivated behavior. ${ }^{70}$ Moreover, activation of mu opioid, endocannabinoid, ${ }^{71}$ and GABA-A receptors in the NAc may mediate the hedonic perception of rewards. ${ }^{72} \mathrm{~A}$ reduced GABA concentration in the prefrontal cortex has been specifically associated with depression and high anhedonia scores. ${ }^{73}$ Serotonin $(5-\mathrm{HT})$ originating from the raphe nuclei and modulating dopamine and opioid release ${ }^{74}$ may also regulate reward-related processes. For example, chronic serotonin-noradrenaline reuptake inhibitor antidepressant treatment is reported to increase ventral striatal activity in patients with depression, ${ }^{75}$ and behavioral results in healthy subjects showed that a selective serotonin reuptake inhibitor (SSRI) treatment improved global performance to obtain a reward, through specific diminution of the effort cost, highlighting the role of serotonin in behavioral regulation. ${ }^{76}$ The abovementioned description of neurotransmitters is not exhaustive but provides some insight on the possible effects of disruptions in the underlying circuits on reward-related processes. 


\section{Clinical implications: assessment and management of reward- related impairments}

As we previously mentioned, reward-related impairments have recently become a growing research interest, as they may persist after remission and lead to a higher risk of poorer treatment response and functional outcomes, such as relapse or recurrence in patients with depression. They may be considered as likely endophenotypes for MDD and have consistent neurobiological substrates; thus, they must be considered a legitimate therapeutic target.

However, there is a lack of consensus on the terminology used to describe these impairments in clinical (symptoms such as amotivation, anhedonia, apathy, avolition, and anergia) and neuropsychological (components such as hedonic capacity or reward responsiveness, reward learning, and motivation or incentive salience or ECDM) domains, possibly explaining the lack of consistent epidemiological data and gold-standard assessment tools in this field. ${ }^{77}$

Nevertheless, assessment and early management of reward-related impairments would be of most benefit to patients with depression and could ultimately lead to personalized treatment. Recent data suggest that the presence of both reward-related symptoms and impairments (eg, reduced reward learning) in individuals with depression predicts a poor outcome with antidepressant treatment, over and above depression severity and other previously reported predictors of outcome or comorbid anxiety. ${ }^{9,24}$ This finding could possibly be related to abnormal cortical-striatal activity and connectivity. ${ }^{11,57}$ Interestingly, in a large prospective study, motivation was the most impaired dimension reported at baseline by patients with depression but had the strongest capacity to detect early improvement; the best predictive value for antidepressant treatment response; and the largest global margin of progress ${ }^{78}$ raising the interest for a special emphasis on reward-related processing within the therapeutic process to improve clinical outcomes. However, treatments may confound the interpretation of findings from reward-related processing studies in MDD, as symptoms of reduced emotion and motivation have been reported as a side-effect of antidepressants (especially SSRI) ${ }^{79}$ and may be worsened by concomitant medication initiation or discontinuation such as benzodiazepines or antipsychotics.

Findings on both biological and nonbiological therapies (eg, behavioral and cognitive remediation or psychotherapy) specifically focusing on reward-related impairment in depression are still lacking. However, cognitive behavioral therapy (CBT) appears to be most beneficial in reducing depressive symptoms for patients with depression who demonstrated decreased reward sensitivity (assessed using ERP) prior to treatment. ${ }^{80}$ Some DBS targets core reward network regions such as the ventral striatum and lateral habenula for treatment of resistant MDD. ${ }^{48,56}$ Lastly, transcranial direct current stimulation (tDCS) targeting the frontopolar cortex enhanced motivation to exert effort for rewards, therefore, representing interesting therapeutic prospects. ${ }^{81}$

In a comprehensive article summarizing the search for a consensus by a group of experts in the optimal approach to study motivation impairments in mood disorders, Calabrese et al (2014) pointed out that there is currently neither a widely accepted gold-standard assessment scale for measuring reward-related impairment (qualified as "amotivation" by the authors) either in isolation or within the context of neuropsychiatric disorders, nor a rating scale defining the clinically relevant effect of treatment on motivation symptoms. ${ }^{77}$ Even if symptoms overlap in different usual depression rating scales (such as Hamilton Depression Scale [HAM-D 17], ${ }^{82}$ Montgomery and Asberg Depression Rating Scale [MADRS], ${ }^{83}$ and $\mathrm{BDI}^{84}$ ), they recommended distinguishing amotivation from other constructs such as fatigue (eg, Fatigue Severity Scale $[\mathrm{FSS}]{ }^{85}$ fatigue visual analog scale [VAS], and Massachusetts General Hospital Cognitive \& Physical Functioning Questionnaire [MGH CPFQ] that measures both amotivation and fatigue ${ }^{86}$ ), anhedonia (eg, SnaithHamilton Anhedonia Pleasure Scale $\left[\mathrm{SHAPS}{ }^{87}\right)$, and cognitive and executive dysfunction (eg, MGH CPFQ). They also recommended assessing the overall impact of reward-related impairment on functional outcomes (eg, Sheehan Disability Scale $\left.[\mathrm{SDS}]^{88}\right)$, quality of life (eg, Quality of Life Enjoyment and Satisfaction Questionnaire $[\mathrm{QLESQ}]^{89}$ ), and distress experienced by caregivers (eg, Apathy Evaluation Scale $[\mathrm{AES}]^{90}$ and Neuropsychiatric Inventory-Apathy subscale [NPI-a $]^{91}$ ).

Some of these scales were used in the studies described in this review, such as the usual depression rating scales; the anhedonia scale, SHAPS; and the apathy scale, AES; some others were used to examine specific aspects of depression/anxiety (eg, Mood and Anxiety Symptom Questionnaire [MASQ $]^{15}$ ), anhedonia (eg, Temporal Experience of Pleasure Scale [TEPS $^{18,19,42}$ ), specific depressive dimensions such as motivation (eg, Multidimensional Assessment of Thymic States scale $[\mathrm{MAThyS}]^{78}$ ), perceived global stress (eg, Perceived Stress Scale $[\mathrm{PST}]^{25}$ ), cognitive symptoms (eg, $\mathrm{MMSE}^{59,62}$ ), and global functioning (eg, Global Assessment of Functioning Scale $[\mathrm{GAF}]^{34}$ ). Relevant measurement tools of reward-related impairments in patients with depression are needed to provide more personalized preventive and therapeutic strategies. 


\section{Reward-Related Processes and Suicidal Behavior}

While the risk of suicidal acts is increased in depression in comparison with the general population, less than $10 \%$ of patients with depression will die from suicide, and less than $50 \%$ will attempt suicide. ${ }^{92}$ Literature now suggests that individuals with a history of depression and suicidal behavior represent a subgroup of patients with particular clinical, cognitive, and biological characteristics. ${ }^{93}$ Among these characteristics, an increasing number of studies have underlined the relevance of reward-related processes in suicidal behavior in the context of depression. ${ }^{94}$

Indirect arguments were first given in the 1970s when low levels of 5-HIAA, the main metabolite of serotonin, were found in patients with depression and a history of violent suicidal acts. ${ }^{95}$ Subsequently, it was found that low 5-HIAA levels were predictive of future death from suicide. ${ }^{96}$ In postmortem studies, individuals who committed suicide had alterations in serotonergic transporter binding in different regions of the prefrontal cortex independently from depression. ${ }^{97}$ The "short" allele of the gene coding for the serotonin transporter (5-HTTLPR) was also associated with an increased risk of suicide attempts, unrelated to alcohol dependence or comorbid depression. ${ }^{98}$ Among many homeostatic functions, serotonin is implicated in the modulation of risky choices through negative outcome-value associative learning and processing of positive reward signals. ${ }^{99}$

More direct evidence for a role of reward-related processes in suicidal behavior was given with the investigation of decision-making. In comparison with patients with a history of depression but no personal history of suicidal behavior and with healthy controls, non-currently depressed suicide attempters showed riskier decision-making during the IGT: in uncertain conditions, attempters preferred options yielding high rewards but even higher losses to options yielding low rewards but lower losses. ${ }^{100}$ Alterations were particularly marked in those who used a violent suicidal means. A meta-analysis of 9 studies in mood disorders confirmed impaired performance in patients with and without a history of suicidal behavior. ${ }^{101}$ These deficits were found in all age groups including the elderly ${ }^{102}$ and adolescents $^{103}$ and were found in attempters, but not in "pure" ideators, ${ }^{104}$ suggesting a particular role in the transition from ideas to acts. Interestingly, impaired decisionmaking was also found in relatives of suicide completers with no personal history of suicidal acts, leading to the idea that it may represent an endophenotype. ${ }^{105}$ Moreover, genetic variants in serotonergic genes were found to modulate decision-making performance in suicide attempters, suggesting a significant role of the serotonergic system, as suspected. ${ }^{106}$ Using functional neuroimaging and the IGT, our group found in both remitted suicide attempters and healthy relatives of suicide victims changes in brain activations according to risk level in dorsal and ventral prefrontal cortex. ${ }^{107-109}$ Using a reversal-learning task in depressed elderly patients, Dombrovski et al showed higher discounting in suicide attempters (but not in ideators) and suggested that attempters tend to overly focus on current outcomes and ignore past experience to guide their choices. ${ }^{110}$ In the same population, neuroimaging was able to differentiate changes associated with depression from those associated with suicidal behavior: a history of a suicide attempt was linked to reduced expected reward signals in the ventromedial prefrontal cortex (in turn predicting insensitivity to contingency changes during decisionmaking), while depression was linked to perturbed encoding of unpredicted rewards in various corticostriatothalamic regions (in turn predicting an oversensitivity to punishment). ${ }^{111}$

Alterations in reward-related processes in suicidal behavior likely extend beyond abstract stimuli (like money) to social situations. For instance, suicide attempters had increased responses to angry, but not happy or sad, faces in the orbitofrontal cortex ${ }^{107,109-112}$ and impaired responses to social exclusion in the posterior insula and parietal cortex. ${ }^{113}$ Both results have been interpreted as an excessive valuation of signals of social reject and disapproval (social punishments). Moreover, elderly suicide attempters tend to reject unfair offers at the Ultimatum Game, whatever the level of unfairness and sacrifice it represents for themselves. ${ }^{114}$ These patients may, therefore, value unfairness excessively, a behavior also observed in individuals submitted to acute tryptophan depletion. ${ }^{115}$

In summary, these findings suggest impaired rewardrelated and valuation processes in individuals at risk of suicidal behavior. It is hypothesized that depression contributes to acute cognitive disturbances that add up to trait deficits (constituting elements of vulnerability) during the suicidal crisis. More research will be necessary to explore the new dimensions of rewardrelated processes (eg, effort costs), dissect the neurocognitive basis of various suicidal phenotypes (eg, violent vs nonviolent suicidal acts and ideas vs acts), make sense of these alterations within the suicidal processes, understand the link with other cognitive deficits observed in suicidal patients (affecting, for example, memory and verbal fluency), and identify therapeutic and preventive tools targeting risk and reward processing.

\section{Conclusion}

Wanting, liking, and learning from/for rewards represent core aspects of MDDs, interestingly, at the border of clinical and neuropsychological approaches. Indeed, 
anhedonia and amotivation could constitute the clinical face of cognitive functions, which help drive behavior adaptively among the different options of reward-related learning. Three core aspects have been distinguished: (1) the ability to represent the rewarding value of a stimulus and establish predictions of future reward to guide and modify behavior to maximize reward (namely reinforcement learning); (2) capacity to assess or react toward pleasantness (namely reward responsiveness, sometimes quoted as liking); and (3) incentive motivation for a reward (namely motivation, sometimes quoted as wanting). Many paradigms are being used (Table 1) that have the advantage to focus on specific aspects of the reward process; however, as the tasks used frequently have overlapping aspects, it is somewhat difficult to see which aspects could be more basic "risk factors" (for example, because shared in at-risk families or having endophenotype properties), which could represent "state-marker" (and be more related to clinical symptoms) and which could constitute "stigmates" of past depressive episodes. However, there is little doubt that assessing these core functions, with comparable and valid instruments, in everyday practice, would be of considerable help to disentangle what could explain unresolved symptoms (incomplete remission), characteristics of patient subgroups (such as highly recurrent and treatment-resistant patients), and risk factors in vulnerable populations (for example, those having familial history of depression). Such practice would have a large impact, as the neurotransmitters involved and brain area in charge are more precisely described, offering new opportunities for personalized treatments.

\section{Disclosure}

Marie-Laure Cléry-Melin and Fabrice Jollant have nothing to disclose. Philip Gorwood reports grants from Ethypharm, grants and personal fees from Servier, personal fees from Janssen, personal fees from Lilly, personal fees from Lundbeck, and personal fees from Otsuka, outside the submitted work.

\section{REFERENCES:}

1. American Psychiatric Association. Diagnostic and statistical manual of mental disorders, 5th ed. Arlington, VA: American Psychiatric Publishing; 2013.

2. Austin MP, Mitchell P, Goodwin GM. Cognitive deficits in depression: possible implications for functional neuropathology. Br J Psychiatry. 2001; 178(03): 200-206.

3. Weingartner H, Cohen RM, Murphy DL, Martello J, Gerdt C. Cognitive processes in depression. Arch Gen Psychiatry. 1981; 38 (1): 42-47.

4. Cléry-Melin ML, Gorwood P. Are cognitive deficits in major depressive disorders progressive? Cambridge: Cambridge University Press; 2016.
5. Treadway MT, Bossaller NA, Shelton RC, Zald DH. Effort-based decision-making in major depressive disorder: a translational model of motivational anhedonia. JAbnorm Psychol. 2012; 121(3): $553-558$.

6. Chen C, Takahashi T, Nakagawa S, Inoue T, Kusumi I. Reinforcement learning in depression: A review of computational research. Neurosci Biobehav Rev. 2015; 55: 247-267.

7. Hélie S, Shamloo F, Novak K, Foti D. The roles of valuation and reward processing in cognitive function and psychiatric disorders. Ann N Y Acad Sci. 2017; 1395(1): 33-48.

8. Must A, Horvath S, Nemeth VL, Janka Z. The Iowa Gambling Task in depression - what have we learned about sub-optimal decisionmaking strategies? Front Psychol. 2013; 4: 732.

9. Uher R, Perlis RH, Henigsberg N, et al. Depression symptom dimensions as predictors of antidepressant treatment outcome: replicable evidence for interest-activity symptoms. Psychol Med. 2012; 42(5): 967-980.

10. Gottesman II, Gould TD. The endophenotype concept in psychiatry: etymology and strategic intentions. Am J Psychiatry. 2003; 160(4): 636-645.

11. Admon R, Pizzagalli DA. Dysfunctional reward processing in depression. Curr Opin Psychol. 2015; 4: 114-118.

12. Berridge KC, Robinson TE, Aldridge JW. Dissecting components of reward: 'liking', 'wanting', and learning. Curr Opin Pharmacol. 2009; 9(1): 65-73.

13. Berridge KC, Kringelbach ML. Neuroscience of affect: brain mechanisms of pleasure and displeasure. Curr Opin Neurobiol. 2013; 23(3): 294-303.

14. Chase HW, Kumar P, Eickhoff SB, Dombrovski AY. Reinforcement learning models and their neural correlates: an activation likelihood estimation meta-analysis. Cogn Affect Behav Neurosci. 2015; 15(2): 435-459.

15. Pizzagalli DA, Iosifescu D, Hallett LA, Ratner KG, Fava M. Reduced hedonic capacity in major depressive disorder: evidence from a probabilistic reward task. J Psychiatr Res. 2008; 43(1): 76-87.

16. O'Doherty JP. Reward representations and reward-related learning in the human brain: insights from neuroimaging. Curr Opin Neurobiol. 2004; 14(6): 769-776.

17. Pessiglione M, Vinckier F, Bouret S, Daunizeau J, Le Bouc R Why not try harder? Computational approach to motivation deficits in neuro-psychiatric diseases. Brain. 2017.

18. Yang XH, Huang J, Zhu CY, et al. Motivational deficits in effortbased decision making in individuals with subsyndromal depression, first-episode and remitted depression patients. Psychiatry Res. 2014; 220(3): 874-882.

19. Yang XH, Huang J, Lan Y, et al. Diminished caudate and superior temporal gyrus responses to effort-based decision making in patients with first-episode major depressive disorder. Prog Neuropsychopharmacol Biol Psychiatry. 2016; 64: 52-59.

20. Bonnelle V, Veromann KR, Burnett Heyes S, Lo Sterzo E, Manohar $\mathrm{S}$, Husain M. Characterization of reward and effort mechanisms in apathy. J Physiol Paris. 2015; 109(1-3): 16-26.

21. Schmidt L, d'Arc BF, Lafargue G, et al. Disconnecting force from money: effects of basal ganglia damage on incentive motivation. Brain. 2008; 131(Pt 5): 1303-1310.

22. Huys QJ, Pizzagalli DA, Bogdan R, Dayan P. Mapping anhedonia onto reinforcement learning: a behavioural meta-analysis. Biol Mood Anxiety Disord. 2013; 3(1): 12.

23. Eshel N, Roiser JP. Reward and punishment processing in depression. Biol Psychiatry. 2010; 68(2): 118-124.

24. Vrieze E, Pizzagalli DA, Demyttenaere K, et al. Reduced reward learning predicts outcome in major depressive disorder. Biol Psychiatry. 2013; 73(7): 639-645. 
25. Pechtel P, Dutra SJ, Goetz EL, Pizzagalli DA. Blunted reward responsiveness in remitted depression. J Psychiatr Res. $2013 ; 47$ (12): 1864-1869.

26. Whitton AE, Kakani P, Foti D, et al. Blunted neural responses to reward in remitted major depression: A high-density event-related potential study. Biol Psychiatry Cogn Neurosci Neuroimaging. 2016; 1(1): 87-95.

27. Rothkirch M, Tonn J, Köhler S, Sterzer P. Neural mechanisms of reinforcement learning in unmedicated patients with major depressive disorder. Brain. 2017; 140(4): 1147-1157.

28. Alloy LB, Olino T, Freed RD, Nusslock R. Role of reward sensitivity and processing in major depressive and bipolar spectrum disorders. Behav Ther. 2016; 47(5): 600-621.

29. Culbreth AJ, Moran EK, Barch DM. Effort-cost decision-making in psychosis and depression: could a similar behavioral deficit arise from disparate psychological and neural mechanisms. Psychol Med. 20171-20216.

30. Pizzagalli DA. Depression, stress, and anhedonia: toward a synthesis and integrated model. Annu Rev Clin Psychol. 2014; 10 (1): 393-423.

31. Cléry-Melin ML, Schmidt L, Lafargue G, Baup N, Fossati P, Pessiglione M. Why don't you try harder? An investigation of effort production in major depression. PLoS One. 2011; 6(8): e23178.

32. Mauras T, Masson M, Fossati P, Pessiglione M. Incentive sensitivity as a behavioral marker of clinical remission from major depressive episode. J Clin Psychiatry. 2016; 77(6): e697-e703.

33. Hershenberg R, Satterthwaite TD, Daldal A, et al. Diminished effort on a progressive ratio task in both unipolar and bipolar depression. J Affect Disord. 2016; 196: 97-100.

34. Sherdell L, Waugh CE, Gotlib IH. Anticipatory pleasure predicts motivation for reward in major depression. J Abnorm Psychol. 2012; 121(1): 51-60.

35. Treadway MT, Buckholtz JW, Schwartzman AN, Lambert WE, Zald DH. Worth the 'EEfRT'? The effort expenditure for rewards task as an objective measure of motivation and anhedonia. PLoS One. $2009 ;$ 4(8): e6598.

36. McIntyre RS, Cha DS, Soczynska JK, et al. Cognitive deficits and functional outcomes in major depressive disorder: determinants, substrates, and treatment interventions. Depress Anxiety. 2013; 30(6): 515-527.

37. Weiland-Fiedler P, Erickson K, Waldeck T, et al. Evidence for continuing neuropsychological impairments in depression. J Affect Disord. 2004; 82(2): 253-258.

38. Pulcu E, Trotter PD, Thomas EJ, et al. Temporal discounting in major depressive disorder. Psychol Med. 2014; 44(9): 1825-1834.

39. Gotlib IH, Hamilton JP, Cooney RE, Singh MK, Henry ML, Joormann J. Neural processing of reward and loss in girls at risk for major depression. Arch Gen Psychiatry. 2010; 67(4): 380-387.

40. McCabe C, Woffindale C, Harmer CJ, Cowen PJ. Neural processing of reward and punishment in young people at increased familial risk of depression. Biol Psychiatry. 2012; 72(7): 588-594.

41. Bress JN, Foti D, Kotov R, Klein DN, Hajcak G. Blunted neural response to rewards prospectively predicts depression in adolescent girls. Psychophysiology. 2013; 50(1): 74-81.

42. Liu WH, Roiser JP, Wang LZ, et al. Anhedonia is associated with blunted reward sensitivity in first-degree relatives of patients with major depression. J Affect Disord. 2016; 190: 640-648.

43. Hasler G, Drevets WC, Manji HK, Charney DS. Discovering endophenotypes for major depression. Neuropsychopharmacology. 2004; 29(10): 1765-1781.

44. Bogdan R, Pizzagalli DA. The heritability of hedonic capacity and perceived stress: a twin study evaluation of candidate depressive phenotypes. Psychol Med. 2009; 39(2): 211-218.

45. Webb CA, Dillon DG, Pechtel P, et al. Neural correlates of three promising endophenotypes of depression: evidence from the
EMBARC Study. Neuropsychopharmacology. 2016; 41(2): 454-463.

46. Haber SN, Knutson B. The reward circuit: linking primate anatomy and human imaging. Neuropsychopharmacology. 2010; 35(1): 4-26.

47. Pizzagalli DA, Holmes AJ, Dillon DG, et al. Reduced caudate and nucleus accumbens response to rewards in unmedicated individuals with major depressive disorder. Am J Psychiatry. 2009; 166(6): 702-710.

48. Nauczyciel C, Robic S, Dondaine T, et al. The nucleus accumbens: a target for deep brain stimulation in resistant major depressive disorder. J Mol Psychiatry. 2013; 1(1): 17.

49. Deng W, Rolls ET, Ji X, et al. Separate neural systems for behavioral change and for emotional responses to failure during behavioral inhibition. Hum Brain Mapp. 2017.

50. O'Doherty JP. The problem with value. Neurosci Biobehav Rev. 2014; 43: 259-268.

51. Admon R, Nickerson LD, Dillon DG, et al. Dissociable corticostriatal connectivity abnormalities in major depression in response to monetary gains and penalties. Psychol Med. 2015; 45(1): 121-131.

52. Der-Avakian A, Markou A. The neurobiology of anhedonia and other reward-related deficits. Trends Neurosci. 2012; 35(1): 68-77.

53. Knutson B, Bhanji JP, Cooney RE, Atlas LY, Gotlib IH. Neural responses to monetary incentives in major depression. Biol Psychiatry. 2008; 63(7): 686-692.

54. Delgado MR. Reward-related responses in the human striatum. Ann N Y Acad Sci. 2007; 1104(1): 70-88.

55. Liu WH, Valton V, Wang LZ, Zhu YH, Roiser JP. Association between habenula dysfunction and motivational symptoms in unmedicated major depressive disorder. Soc Cogn Affect Neurosci. 2017; 12(9): 1520-1533.

56. Morishita T, Fayad SM, Higuchi MA, Nestor KA, Foote KD. Deep brain stimulation for treatment-resistant depression: systematic review of clinical outcomes. Neurotherapeutics. 2014; 11(3): 475-484.

57. Ubl B, Kuehner C, Kirsch P, Ruttorf M, Diener C, Flor H. Altered neural reward and loss processing and prediction error signalling in depression. Soc Cogn Affect Neurosci. 2015; 10(8): 1102-1112.

58. Admon R, Kaiser RH, Dillon DG, et al. Dopaminergic enhancement of striatal response to reward in major depression. Am J Psychiatry. 2017; 174(4): 378-386.

59. Gong L, Yin Y, He C, et al. Disrupted reward circuits is associated with cognitive deficits and depression severity in major depressive disorder. J Psychiatr Res. 2017; 84: 9-17.

60. Kaiser RH, Andrews-Hanna JR, Wager TD, Pizzagalli DA. Largescale network dysfunction in major depressive disorder: a metaanalysis of resting-state functional connectivity. JAMA Psychiatry. 2015; 72(6): 603-611.

61. Menon V. Large-scale brain networks and psychopathology: a unifying triple network model. Trends Cogn Sci. 2011; 15(10): 483-506.

62. Quevedo $\mathrm{K}, \mathrm{Ng} \mathrm{R}$, Scott $\mathrm{H}$, et al. Ventral striatum functional connectivity during rewards and losses and symptomatology in depressed patients. Biol Psychol. 2017; 123: 62-73.

63. Folstein MF, Folstein SE, McHugh PR. "Mini-mental state". A practical method for grading the cognitive state of patients for the clinician. J Psychiatr Res. 1975; 12(3): 189-198.

64. Sharma A, Wolf DH, Ciric R, et al. Common dimensional reward deficits across mood and psychotic disorders: a connectome-wide association study. Am J Psychiatry. 2017; 174(7): 657-666.

65. Park IH, Lee BC, Kim JJ, Kim JI, Koo MS. Effort-based reinforcement processing and functional connectivity underlying amotivation in medicated patients with depression and schizophrenia. J Neurosci. 2017; 37(16): 4370-4380. 
66. Liu WH, Wang LZ, Shang HR, et al. The influence of anhedonia on feedback negativity in major depressive disorder. Neuropsychologia. 2014; 53: 213-220.

67. Foti D, Carlson JM, Sauder CL, Proudfit GH. Reward dysfunction in major depression: multimodal neuroimaging evidence for refining the melancholic phenotype. Neuroimage. 2014; 101: 5058.

68. Gorwood P. Neurobiological mechanisms of anhedonia. Dialogues Clin Neurosci. 2008; 10(3): 291-299.

69. Skolnick P, Popik P, Trullas R. Glutamate-based antidepressants: 20 years on. Trends Pharmacol Sci. 2009; 30(11): 563-569.

70. Stuber GD, Sparta DR, Stamatakis AM, et al. Excitatory transmission from the amygdala to nucleus accumbens facilitates reward seeking. Nature. 2011; 475(7356): 377-380.

71. Berridge KC, Kringelbach ML. Affective neuroscience of pleasure: reward in humans and animals. Psychopharmacology (Berl). 2008; 199(3): 457-480.

72. Faure A, Richard JM, Berridge KC. Desire and dread from the nucleus accumbens: cortical glutamate and subcortical GABA differentially generate motivation and hedonic impact in the rat. PLoS One. 2010; 5(6): e11223.

73. Gabbay V, Mao X, Klein RG, et al. Anterior cingulate cortex $\gamma$ aminobutyric acid in depressed adolescents: relationship to anhedonia. Arch Gen Psychiatry. 2012; 69(2): 139-149.

74. Yan QS. Activation of 5-HT2A/2C receptors within the nucleus accumbens increases local dopaminergic transmission. Brain Res Bull. 2000; 51(1): 75-81.

75. Ossewaarde L, Verkes RJ, Hermans EJ, et al. Two-week administration of the combined serotonin-noradrenaline reuptake inhibitor duloxetine augments functioning of mesolimbic incentive processing circuits. Biol Psychiatry. 2011; 70(6): 568-574.

76. Meyniel F, Goodwin GM, Deakin JW, et al. A specific role for serotonin in overcoming effort cost. eLife. 2016; 5: 5 .

77. Calabrese JR, Fava M, Garibaldi G, et al. Methodological approaches and magnitude of the clinical unmet need associated with amotivation in mood disorders. J Affect Disord. 2014; 168: 439-451.

78. Gorwood P, Vaiva G, Corruble E, Llorca PM, Baylé FJ, Courtet P. The ability of early changes in motivation to predict later antidepressant treatment response. Neuropsychiatr Dis Treat. 2015; 11: 2875-2882.

79. Price J, Cole V, Goodwin GM. Emotional side-effects of selective serotonin reuptake inhibitors: qualitative study. Br J Psychiatry. 2009; 195(3): 211-217.

80. Burkhouse KL, Kujawa A, Kennedy AE, et al. Neural reactivity to rewards as a predictor of cognitive behavioral therapy response in anxiety and depression. Depress Anxiety. 2016; 33(4) 281-288.

81. Soutschek A, Kang P, Ruff CC, et al. Brain stimulation over the frontopolar cortex enhances motivation to exert effort for reward. Biol Psychiatry. 2017.

82. Hamilton M. A rating scale for depression. J Neurol Neurosurg Psychiatry. 1960; 23(1): 56-62.

83. Montgomery SA, Asberg M. A new depression scale designed to be sensitive to change. Br J Psychiatry. 1979; 134(4): 382-389.

84. Beck AT, Ward CH, Mendelson M, Mock J, Erbaugh J. An inventory for measuring depression. Arch Gen Psychiatry. 1961; 4 (6): 561-571.

85. Demyttenaere K, De Fruyt J, Stahl SM. The many faces of fatigue in major depressive disorder. Int J Neuropsychopharmacol. 2005; 8 (1): 93-105.

86. Fava M, Iosifescu DV, Pedrelli P, Baer L. Reliability and validity of the Massachusetts general hospital cognitive and physical functioning questionnaire. Psychother Psychosom. 2009; 78(2): 91-97.
87. Snaith RP, Hamilton M, Morley S, Humayan A, Hargreaves D, Trigwell P. A scale for the assessment of hedonic tone the Snaith-Hamilton Pleasure Scale. Br J Psychiatry. 1995; 167(1): 99-103.

88. Sheehan DV, Harnett-Sheehan K, Spann ME, Thompson HF, Prakash A. Assessing remission in major depressive disorder and generalized anxiety disorder clinical trials with the discan metric of the Sheehan disability scale. Int Clin Psychopharmacol. 2011; 26 (2): 75-83.

89. Hope ML, Page AC, Hooke GR. The value of adding the Quality of Life Enjoyment and Satisfaction Questionnaire to outcome assessments of psychiatric inpatients with mood and affective disorders. Qual Life Res. 2009; 18(5): 647-655.

90. Marin RS, Biedrzycki RC, Firinciogullari S. Reliability and validity of the Apathy Evaluation Scale. Psychiatry Res. 1991; 38(2): 143-162.

91. Cummings JL, Mega M, Gray K, Rosenberg-Thompson S, Carusi DA, Gornbein J. The Neuropsychiatric Inventory: comprehensive assessment of psychopathology in dementia. Neurology. 1994; 44 (12): 2308-2314.

92. Isometsä E. Suicidal behaviour in mood disorders-who, when, and why? Can J Psychiatry. 2014; 59(3): 120-130.

93. Turecki G, Brent DA. Suicide and suicidal behaviour. Lancet. 2016 387(10024): 1227-1239.

94. Jollant F, Lawrence NL, Olié E, Guillaume S, Courtet P. The suicidal mind and brain: a review of neuropsychological and neuroimaging studies. World J Biol Psychiatry. 2011; 12(5): 319339.

95. Asberg M, Träskman L, Thorén P. 5-HIAA in the cerebrospinal fluid. A biochemical suicide predictor? Arch Gen Psychiatry. 1976; 33(10): 1193-1197.

96. Mann JJ, Currier D, Stanley B, Oquendo MA, Amsel LV, Ellis SP. Can biological tests assist prediction of suicide in mood disorders? Int J Neuropsychopharmacol. 2006; 9(4): 465-474.

97. Mann JJ, Huang YY, Underwood MD, et al. A serotonin transporter gene promoter polymorphism (5-HTTLPR) and prefrontal cortical binding in major depression and suicide. Arch Gen Psychiatry. 2000; 57(8): 729-738.

98. Gorwood P, Batel P, Adès J, Hamon M, Boni C. Serotonin transporter gene polymorphisms, alcoholism, and suicidal behavior. Biol Psychiatry. 2000; 48(4): 259-264.

99. Rogers RD. The roles of dopamine and serotonin in decision making: evidence from pharmacological experiments in humans. Neuropsychopharmacology. 2011; 36(1): 114-132.

100. Jollant F, Bellivier F, Leboyer M, et al. Impaired decision making in suicide attempters. Am J Psychiatry. 2005; 162(2): 304-310.

101. Richard-Devantoy S, Berlim MT, Jollant F. A meta-analysis of neuropsychological markers of vulnerability to suicidal behavior in mood disorders. Psychol Med. 2014; 44(8): 1663-1673.

102. Clark L, Dombrovski AY, Siegle GJ, et al. Impairment in risksensitive decision-making in older suicide attempters with depression. Psychol Aging. 2011; 26(2): 321-330.

103. Bridge JA, McBee-Strayer SM, Cannon EA, et al. Impaired decision making in adolescent suicide attempters. J Am Acad Child Adolesc Psychiatry. 2012; 51(4): 394-403.

104. Sheftall AH, Davidson DJ, McBee-Strayer SM, et al. Decisionmaking in adolescents with suicidal ideation: A case-control study Psychiatry Res. 2015; 228(3): 928-931.

105. Hoehne A, Richard-Devantoy S, Ding Y, Turecki G, Jollant F. Firstdegree relatives of suicide completers may have impaired decisionmaking but functional cognitive control. J Psychiatr Res. 2015; 68 192-197.

106. Jollant F, Buresi C, Guillaume S, et al. The influence of four serotonin-related genes on decision-making in suicide 
attempters. Am J Med Genet B Neuropsychiatr Genet. 2007; 144B (5): 615-624.

107. Ding Y, Pereira F, Hoehne A, et al. Altered brain processing of decision-making in healthy first-degree biological relatives of suicide completers. Mol Psychiatry. 2017; 22(8): 1149-1154.

108. Jollant F, Lawrence NS, Olie E, et al. Decreased activation of lateral orbitofrontal cortex during risky choices under uncertainty is associated with disadvantageous decision-making and suicidal behavior. Neuroimage. 2010; 51(3): 1275-1281.

109. Olié E, Ding Y, Le Bars E, et al. Processing of decision-making and social threat in patients with history of suicidal attempt: A neuroimaging replication study. Psychiatry Res. 2015; 234(3): 369-377.

110. Dombrovski AY, Clark L, Siegle GJ, et al. Reward/punishment reversal learning in older suicide attempters. Am J Psychiatry. 2010; 167(6): 699-707.
111. Dombrovski AY, Szanto K, Clark L, Reynolds CF, Siegle GJ. Reward signals, attempted suicide, and impulsivity in late-life depression. JAMA Psychiatry. 2013; 70(10): 1.

112. Jollant F, Lawrence NS, Giampietro V, et al. Orbitofrontal cortex response to angry faces in men with histories of suicide attempts. Am J Psychiatry. 2008; 165(6): 740-748.

113. Olié E, Jollant F, Deverdun J, et al. The experience of social exclusion in women with a history of suicidal acts: a neuroimaging study. Sci Rep. 2017; 7(1): 89.

114. Szanto K, Clark L, Hallquist M, Vanyukov P, Crockett M, Dombrovski AY. The cost of social punishment and high-lethality suicide attempts in the second half of life. Psychol Aging. 2014; 29 (1): 84-94.

115. Young SN. The effect of raising and lowering tryptophan levels on human mood and social behaviour. Philos Trans R Soc Lond B Biol Sci. $2013 ; \mathbf{3 6 8}(1615)$ : 20110375. 